\title{
Universiteit
}

Leiden

The Netherlands

\section{Turcs et Marocains aux Pays-Bas pendant la Révolution sexuelle (1964-1979) Une analyse photographique} Shield, A.D.J.

\section{Citation}

Shield, A. D. J. (2020). Turcs et Marocains aux Pays-Bas pendant la Révolution sexuelle (1964-1979): Une analyse photographique. Clio. Femmes, Genre, Histoire, 51, 229-239. doi:10.4000/clio.18296
Version:
Publisher's Version
License:
Licensed under Article 25fa Copyright Act/Law (Amendment Taverne)
Downloaded from: $\quad$ https://hdl.handle.net/1887/3200810

Note: To cite this publication please use the final published version (if applicable). 


\section{TURCS ET MAROCAINS AUX PAYS-BAS PENDANT LA RÉVOLUTION SEXUELLE (1964-1979). UNE ANALYSE PHOTOGRAPHIQUE}

Andrew DJ Shield, Traduit de l'anglais par Linda Guerry

Belin | « Clio. Femmes, Genre, Histoire »

2020/1 n $51 \mid$ pages 229 à 239

ISSN 1252-7017

ISBN 9782410017090

DOI 10.4000/clio.18296

Article disponible en ligne à l'adresse :

https://www.cairn.info/revue-clio-femmes-genre-histoire-2020-1-page-229.htm

Distribution électronique Cairn.info pour Belin.

(C) Belin. Tous droits réservés pour tous pays.

La reproduction ou représentation de cet article, notamment par photocopie, n'est autorisée que dans les limites des conditions générales d'utilisation du site ou, le cas échéant, des conditions générales de la licence souscrite par votre établissement. Toute autre reproduction ou représentation, en tout ou partie, sous quelque forme et de quelque manière que ce soit, est interdite sauf accord préalable et écrit de l'éditeur, en dehors des cas prévus par la législation en vigueur en France. Il est précisé que son stockage dans une base de données est également interdit. 


\section{Clio. Femmes, Genre, Histoire}

$51 \mid 2020$

Femmes et genre en migration

\section{Turcs et Marocains aux Pays-Bas pendant la Révolution sexuelle (1964-1979). Une analyse photographique}

Turks and Moroccans in the Netherlands during the sexual revolution

(1964-1979): a photographic analysis

\section{Andrew DJ Shield}

Traducteur : Linda Guerry

\section{OpenEdition}

\section{Journals}

Édition électronique

URL : http://journals.openedition.org/clio/18296

DOI : $10.4000 /$ clio. 18296

ISSN : 1777-5299

Éditeur

Belin

Édition imprimée

Date de publication : 1 juillet 2020

Pagination : 229-239

ISBN : $978-2410017090$

ISSN : 1252-7017

Distribution électronique Cairn

\section{CAIRN, INFO}

CHERCHER, REPÉRER, AVANCER.

\section{Référence électronique}

Andrew DJ Shield, «Turcs et Marocains aux Pays-Bas pendant la Révolution sexuelle (1964-1979). Une analyse photographique », Clio. Femmes, Genre, Histoire [En ligne], 51 | 2020, mis en ligne le 01 janvier 2024, consulté le 24 juin 2020. URL : http://journals.openedition.org/clio/18296 ; DOI : https:// doi.org/10.4000/clio.18296 


\section{Turcs et Marocains aux Pays-Bas pendant la Révolution sexuelle (1964-1979). Une analyse photographique}

De 1965 à 1975, des travailleurs immigrés ${ }^{1}$ venus seuls - la plupart originaires de Turquie, du Maroc et de l'Europe du Sud -, ont participé aux transformations des cultures sexuelles et de genre de l'Europe du Nord, par exemple en fréquentant des espaces hétérosociaux chargés d'érotisme, comme les discothèques, ou en ayant des relations occasionnelles avec des femmes européennes. Cependant, à partir du milieu des années 1970, l'arrivée des épouses et enfants venus de leur pays d'origine conduit à un changement d'attitude de ces hommes à l'égard de la «liberté sexuelle $»^{2}$. Cet article explore, dans le cas des Pays-Bas, ce tournant conservateur chez les travailleurs immigrés lié au regroupement familial. Il propose l'utilisation de nouvelles sources, comme le fonds de collections photographiques déposées par des immigrant.es à l'Institut d'histoire sociale d'Amsterdam $(\mathrm{HBM})^{3}$, et montre l'intérêt de ces archives personnelles pour écrire une histoire de la sexualité et de l'immigration.

1 Comme en Allemagne, le terme néerlandais signifie «travailleurs invités » et Andrew Shield le met entre guillemets. Nous le traduisons par «travailleurs immigrés » et supprimons les guillemets [Ndt].

2 Cet article développe mes analyses sur le genre, la migration et la sociabilité dans les années 1960-1980 développées dans Shield 2017 : 74-81 et 249-253.

3 Historisch Beeldarchief Migranten de l'International Institute of Social History à Amsterdam. 


\section{4-1975 : une fréquentation hétérosociale}

Les traités bilatéraux qui organisent les migrations de travail entre les Pays-Bas et la Turquie (1964), les Pays-Bas et le Maroc (1969), entre autres pays, ont conduit à l'arrivée de plus de 150000 étrangers en 19774. Venus pour combler les besoins du marché du travail dans le contexte de développement économique d'après-guerre, leur expérience aux Pays-Bas est semblable à celle des nombreux travailleurs immigrés dans les pays voisins, comme l'Allemagne ${ }^{5}$. Du recrutement jusqu’à la crise pétrolière de 1973, la grande majorité des hommes immigrés sont célibataires ou bien leurs épouses sont restées dans leur pays d'origine. Pour ces hommes, ces premières années sont l'occasion d'explorer avec curiosité les villes européennes et leurs sous-cultures. Certains hommes fréquentent les discothèques et ont des relations sexuelles occasionnelles avec des Européennes dans un contexte de tournant féministe et de libération sexuelle où de nombreuses femmes affirment leur indépendance socio-économique ou sexo-politique.

Il est difficile de trouver des sources témoignant des désirs et des comportements sexuels des travailleurs immigrés. En 1970, une chercheuse en sciences sociales, qui a étudié les travailleurs turcs au Danemark, note que même ceux prêts à évoquer des «questions personnelles » parlent peu de leurs occupations durant leur temps libre. Elle attribue cette gêne à «des conflits de valeurs, puisque beaucoup d'entre eux font des choses qu'ils ne feraient pas chez eux, comme aller danser ${ }^{6}$. Le fonds d'archives HBM contient des photographies des activités de temps libre des travailleurs immigrés, tout comme deux livres clés portant sur la période analysée; cependant les légendes des photographies sont souvent imprécises ${ }^{7}$. Une photo représentant un homme entouré de plusieurs amis (hommes, turcs) devant la gare centrale d'Amsterdam en 1964 et classée dans la catégorie "activités de loisir (non organisées)», est décrite ainsi par celui qui est sur la photographie: «Au début, je ne faisais pas grand-chose de mon temps libre, à part visiter

\footnotetext{
4 Shadid $1979: 47$.

5 Chin 2007.

6 Mizrahi Sirazi [Mirdal] 1970.

7 Tuskan \& Vogel 2004 ; Cotaar \& Bouras 2009.
} 
Amsterdam... J'étais très curieux de connaittre ce que cette ville avait à offrir $»^{8}$. Sur une autre photographie, un homme turc (arrivé aux Pays-Bas en 1965) pose en 1972 à Copenhague, devant la devanture d'un vidéo-club spécialisé dans la pornographie, à côté d'une affiche représentant une femme seins nus. La légende indique qu'il s'agit d'une des nombreuses fois où l'homme est parti avec des amis visiter « une autre ville européenne $»^{9}$. Le fait que cet homme ait gardé et donné cette photographie suggère qu'il n'était pas opposé à l'affichage de l'érotisme dans l'espace public européen.

Les relations interethniques pendant les premières années de présence des travailleurs immigrés en Europe apparaissent dans les données démographiques et dans les médias des années 1970. Par exemple, un article paru dans le Leeuwarder Courant, rapporte qu'aux Pays-Bas, en 1970, environ un cinquième des grossesses de mères célibataires (2 535 au total) implique un père étranger ${ }^{10}$. L'Association néerlandaise pour la réforme sexuelle (Nederlandse Vereniging voor Seksuele Hervorming - NVSH) propose même au gouvernement de subventionner les visites des bordels pour les travailleurs étrangers ; cette demande est faite au nom du droit de l'individu à la satisfaction sexuelle, mais avec l'idée que la frustration sexuelle pourrait affecter d'autres aspects du bien-être psychologique des immigrants ${ }^{11}$. Cette initiative du NVSH ne peut cependant être dissociée du climat d'anxiété lié aux rencontres interethniques. En effet, au début des années 1970, le déclenchement de plusieurs émeutes raciales (par exemple à Rotterdam) est attribué au sentiment de menace ressenti par des hommes européens suite à des relations interethniques avec des femmes néerlandaises ${ }^{12}$. En 1976, environ un tiers des Marocains dits de la «deuxième génération»(1 133 sur 3 855) ont un parent

8 Tuskan \& Vogel 2004 : 146. L'information bibliographique complète avec les noms des personnes turques qui ont fait don de leur collection de photographies est répertoriée dans Tuskan \& Vogel. Afin d'éviter leurs connexions immédiates à cet article par les moteurs de recherche, j'ai choisi de ne pas inclure les noms complets ici ; voir la partie « réflexions critiques » de cet article.

9 Tuskan \& Vogel 2004 : 148.

10 Source originale : Leeuwarder Courant, 3 février 1972 cité par Bouras 2012 : 73.

11 Voir Ben Jelloun 1977.

12 Schrover 2011. 
néerlandais, bien que cette proportion chute fortement pendant les années de regroupement familial ${ }^{13}$.

Des photos du fonds d'archives illustrent bien les amitiés nouées dans les moments de temps libre, au cours des décennies 1960 et 1970, entre les travailleurs immigrés et les Néerlandaises, notamment à la plage, à la piscine, dans les parcs d'attraction, dans les cafés et à l'occasion de dîners. Par exemple, dans la Figure 1, six hommes et deux femmes, plus ou moins dénudé.es, se détendent sur la pelouse ; la légende indiquée dans le fonds HBM précise que l'homme turc qui a donné la photographie est «avec des amis turcs et des petites amies hollandaises » à Amsterdam en 1965, un an après son arrivée à l'âge de 23 ans. Classée dans la catégorie «loisirs», cette photographie montre un après-midi tranquille de bain de soleil. La plupart des personnes sont en maillots de bain ou sous-vêtements, et une des femmes néerlandaises porte un soutien-gorge ou un haut de bikini. Cette photographie questionne: est-ce que le fait de s'asseoir en compagnie mixte sans chemise ni pantalon est une nouveauté pour ces hommes ? Comment ont-ils ressenti le fait de se déshabiller ou de regarder des femmes déshabillées, de manière aussi désinvolte? Le bikini est aussi relativement nouveau aux Pays-Bas et il est peu probable que ces femmes aient eu des mères qui en portaient ${ }^{14}$. Ces hommes s'étaient-ils déjà baignés aux côtés d'une femme en bikini? Cette photographie laisse un certain nombre de questions ouvertes sur les relations entre ces femmes et ces hommes ou sur le degré de différence entre ce que ces hommes ont vécu cet après-midi-là et ce qu'ils avaient expérimenté en Turquie. Malgré ces inconnues, cette photographie témoigne que, dans les années 1960, des travailleurs étrangers originaires de pays à majorité musulmane étaient ouverts à l'idée de relations avec les Néerlandaises dans des cadres hétérosociaux, voire de flirts.

13 Shield $2017: 69$. Source originale : Centraal Bureau voor Statistiek.

14 Allyn $2000: 28$. 


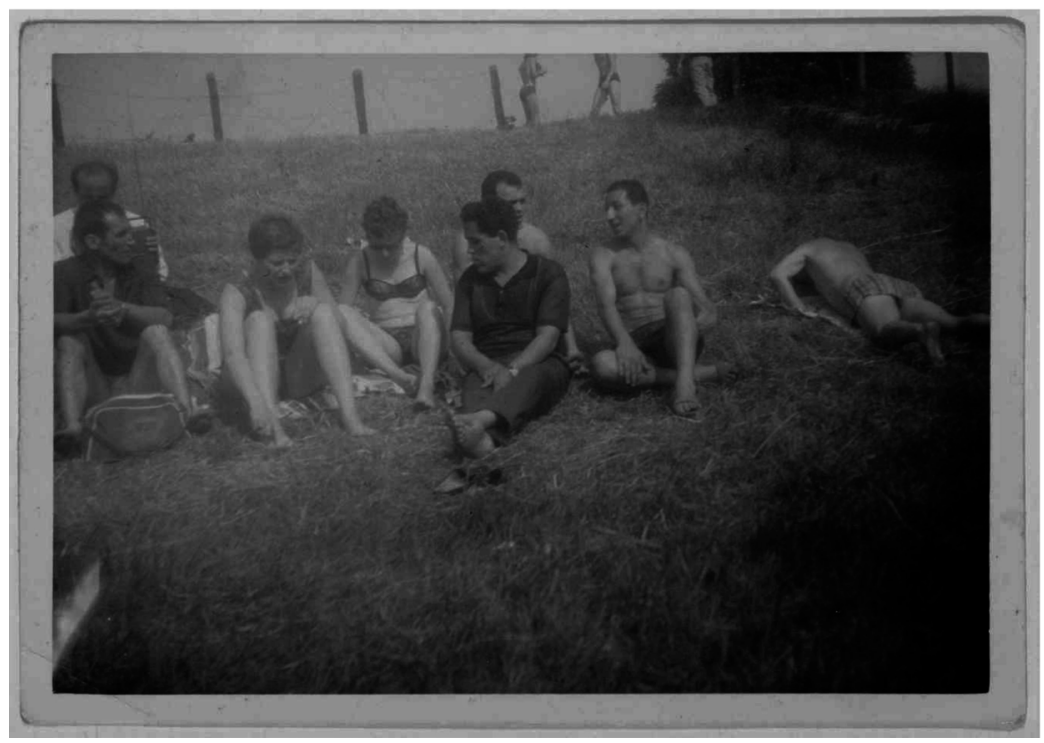

Fig. 1 « Avec des amis turcs et des petites amies hollandaises », Amsterdam 1965 (C) Fonds HBM)

Une autre photographie du fonds HBM montre «les premiers travailleurs turcs à Assen s'amusant dans une piscine» en 1965, l'année où l'homme qui en a fait don est arrivé aux Pays-Bas à l'âge de 26 ans. Pour la photographie de groupe, dix hommes et une femme se serrent les uns contre les autres en maillot de bain. Au centre de l'image, un homme se tient sur les épaules d'un autre. Une femme blonde se serre contre le groupe, touchant légèrement l'homme à côté d'elle ; en dehors du cadre, on devine une deuxième femme assise, enveloppée dans une serviette. Il n'y a aucune mention des femmes dans la légende. Pourtant, sur une autre photographie de ce groupe, également prise dans une auberge à Assen en 1965, on voit dix hommes turcs avec deux femmes blondes. La légende précise que les deux femmes, dont l'une s'appelle Petra, "préparaient le petit déjeuner et changeaient les draps » pour les hommes de l'auberge. Le fait que ces femmes apparaissent dans le portrait de groupe suggère que les hommes se sont liés à elles, et les ont peut-être invitées à des sorties de week- 
end. L'un des hommes turcs semble enrouler affectueusement son bras autour de Petra, mais peut-être son bras est-il seulement posé sur la chaise derrière elle sans qu'il la touche. Cette photographie permet d'entrevoir la possibilité de relations sexuelles interethniques occasionnelles au cours de ces premières années aux Pays-Bas.

\section{6-1979 : regroupement familial et nouveaux loisirs}

Après la crise pétrolière de 1973 et les restrictions qui suivent dans le recrutement de main-d'œuvre, les travailleurs étrangers réalisent dans le recrutement que s'ils quittent l'Europe de l'Ouest pour retourner dans leur pays, ils ne pourront pas y revenir. C'est pourquoi beaucoup d'entre eux décident de faire venir leur épouse dans le pays d'accueil ou d'épouser une femme de leur pays d'origine. Les femmes ont joué un rôle important dans ce processus en faisant pression sur les hommes pour organiser leur immigration. L'historienne Nadia Bouras, qui a mené des dizaines d'entretiens avec les «pionniers » marocains aux Pays-Bas ${ }^{15}$ résume le tournant conservateur des comportements masculins pendant les années de regroupement familial :

Dans les entretiens, les hommes de cette première génération racontent souvent qu'avec l'arrivée de la famille, la misère a commencé. Ils avaient beaucoup de liberté avant la venue de leur femme: ils buvaient de l'alcool, avaient des petites amies. Quand ils retournaient au Maroc, ils étaient les rois. Avec l'arrivée de la famille, ils ont dû prendre le rôle de père et souvent se sont retrouvés au chômage. Les contacts avec la population néerlandaise se sont faits plus rares, ils ont commencé à constituer entre eux un des piliers de la société, à rencontrer d'autres hommes à la mosquée. Les Néerlandais et les Marocains vivaient désormais chacun de leur côté au lieu de vivre ensemble.

Selon Nadia Bouras, et c'est important de le souligner, ce tournant conservateur n'est pas seulement lié à l'arrivée des épouses et des enfants ; il est aussi une réaction à la perte d'emploi et à la précarité économique. Néanmoins, d'autres sources relient ce changement à la réunification familiale. Par exemple, dans l'entretien que j'ai mené avec un Turc qui est venu aux Pays-Bas en tant que «travailleur immigré », celui-ci décrit ainsi le tournant conservateur :

15 Bouras 2019. 
Quand ils sont venus en Hollande, la liberté était partout... Leur femme était à la maison et ils pouvaient faire ce qu'ils voulaient... Mais il y a eu un conflit quand les familles sont venues et cela a totalement changé à la fin des années 1970 et dans les années 1980. [Ceux qui buvaient et dansaient] vingt ans plus tôt, se retrouvent à diriger une mosquée, à devenir très religieux, tout a changé ${ }^{16}$.

Dans un autre entretien, un Marocain, né en 1969 et venu aux PaysBas en 1974, est du même avis; en regardant de vieilles photos de famille, l'homme décrit son père et ses amis comme étant «très modernes et libérés ", mais il a eu l'impression que leurs comportements avaient changé «soudainement... quand ils ont fait venir leurs femmes ici ${ }^{17}$. Des chercheur.es du Dutch Institute for Social Research ont affirmé que les personnes d'origines marocaine et turque attachent aujourd'hui beaucoup plus d'importance à la famille et à la religion que dans les années 1960 et 1970 et que ce « changement de valeurs » a conduit au déclin d'une attitude libérale à l'égard de la sexualité ${ }^{18}$. Les photographies d'immigrant.es suggèrent en effet un changement dans les activités de loisirs des hommes à partir du milieu des années 1970. De toute évidence, il y a plus d'activités familiales pendant et après la réunification familiale; les photographies de cérémonies et de célébrations religieuses sont également nombreuses dans un contexte de développement des institutions musulmanes.

La Figure 2 illustre la constitution d'une communauté diasporique aux Pays-Bas, où trois familles turques se sont réunies pour un repas à Gouda en 1976. Les trois pères portent des moustaches, que peu d'hommes turcs portaient sur les photographies des années 1960, et les trois femmes turques ont les cheveux à moitié couverts. Neuf enfants, presque tous âgés de moins de cinq ans, sont rassemblés devant la table. L'ambiance de la photographie est gaie et festive. Probablement prise avec un retardateur sur un trépied, la photographie montre une atmosphère joyeuse et chaotique où quinze

16 Shield 2017 : 54 et 75 ; source originale : entretien [nom expurgé] d'octobre 2014. Texte original en anglais.

17 Shield 2017 : 76; source originale : entretien [nom expurgé], d'octobre 2014. Texte original en anglais.

18 Keuzenkamp $2010: 362$. 
personnes tentent de poser avant le flash de l'appareil. La photographie saisit le rire d'une mère et l'agitation de la plupart des enfants qui n'arrivent pas à arrêter leurs activités pour la pose. Cette photo du dîner est imprimée sur la dernière page de l'ouvrage Lied nit den vreemde [Chanson de l'étranger] et illustre, pour les travailleurs immigrés, la fin de l'histoire des années pionnières. En parcourant les 220 pages du livre, le lecteur se familiarise avec le recrutement des travailleurs, leur voyage en Europe, leur vie professionnelle et leur logement, ainsi que leurs activités de loisirs. Cette aventure culmine avec l'arrivée de l'épouse et d'enfants, et la construction d'une communauté diasporique aux Pays-Bas.

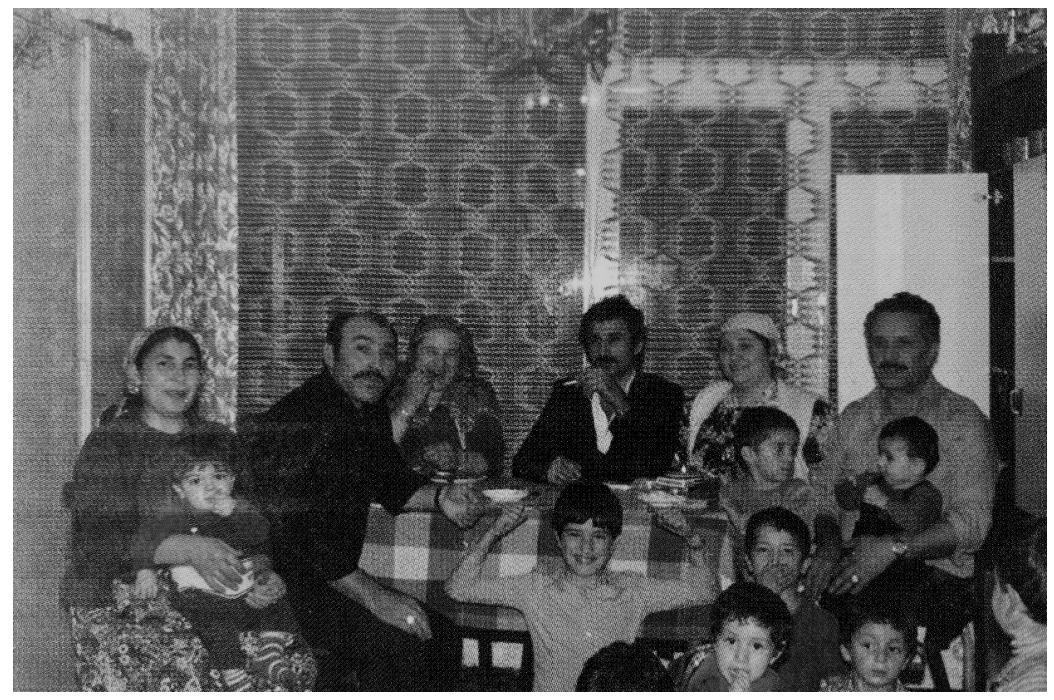

Fig. 2. Trois familles réunies pour un repas à Gouda, 1976 (C) Fonds HBM)

\section{Réflexions critiques}

Les immigrants qui se sont installés dans le nord-ouest de l'Europe à la fin des années 1960 ont participé à certains aspects de la « révolution sexuelle »-sciemment ou non à l'époque - en adoptant une attitude libérale à l'égard de la sexualité, également nouvelle pour 
de nombreux Européens, comme la pratique de relations sexuelles prémaritales occasionnelles ou la fréquentation des premiers magasins légalisés de pornographie. Ces pionniers, désignés comme "travailleurs invités", ont eu tendance à rester vagues dans les entretiens sur leurs activités de loisir au cours de ces premières années dans un nouveau pays. Toutefois, les collections de photographies illustrent bien leur fréquentation de femmes locales et parfois la pratique du flirt. Le contraste de ces images avec les photographies des albums de famille de la décennie suivante illustre le tournant conservateur qui a concerné de nombreux hommes dans leurs attitudes d'hétérosociabilité interethnique. Mais ce «tournant» n'a jamais été complet et l'on pourrait aussi utiliser ces photographies pour nuancer ou contester la notion de «conservatisme». Les enquêtes de la fin des années 1970 montrent que la plupart des hommes marocains installés aux Pays-Bas ne s'opposent pas à ce que leur épouse prenne un emploi à l'extérieur du foyer et adopte des "vêtements de style européen ». Il est donc important de souligner que, comme dans le cas de la population européenne locale, les attitudes des immigrants à l'égard des rapports de genre et de la sexualité changent continuellement au fil du temps.

Avant de conclure sur les désirs et comportements sexuels des personnes, l'historien.ne doit considérer la question d'un point de vue éthique. Dans l'idéal, avant d'affirmer certains propos, l'historien.ne doit confronter les sources et les croiser avec des entretiens et des lettres par exemple. Mais la sexualité est une affaire profondément intime, davantage qu'un album photo de famille. Ceux qui ont fait don de leur collection de photographies que les chercheur.es peuvent utiliser doivent être considérés avec respect. Bien sûr, les historien.nes ne doivent pas utiliser les sources sans questionner d'autres dimensions que leur intention première. Spécialiste des archives coloniales néerlandaises, Ann Stoler soutient que les documents coloniaux ne décrivent pas seulement les politiques et les pratiques, mais disent aussi l'«anxiété profonde de leurs auteurs au sujet de l'ordre colonial ${ }^{19}$. Cependant, contrairement aux archives coloniales, le HBM a été créé pour archiver l'histoire d'un groupe marginalisé

19 Stoler 2010. 
dont les récits pourraient être oubliés par l'histoire européenne. Le contexte sociopolitique actuel compte : ceux qui ont été les pionniers de l'émigration de pays à majorité musulmane, et leurs descendant.es en Europe, sont au cœur d'une rhétorique diffamatoire depuis des décennies. Dans ce contexte, les chercheur.es ont le devoir d'aborder les sujets sensibles, comme la sexualité, avec respect.

Les recherches futures devraient nuancer davantage la corrélation entre l'arrivée des femmes immigrantes et le tournant conservateur. S'il est vrai que ce dernier correspond à l'essor de l'immigration féminine et qu'il est lié au développement des institutions et pratiques musulmanes en Europe du Nord-Ouest, il est aussi la conséquence d'autres facteurs externes, comme le chômage, et le rejet de la libération sexuelle par une partie importante de la population locale dans toute l'Europe du NordOuest au cours des années 1980.

Traduit de l'anglais par Linda GUERRY

\section{Bibliographie}

AlLYN David, 2000, Make Love, Not War: the sexual revolution, an unfettered bistory, Boston, Little, Brown and Company.

Ben Jelloun Tahar, 1977, La Plus Haute des solitudes, Paris, Le Seuil.

BOURAS Nadia, 2012, «Het land van herkomst: perspectieven op verbondenheid met Marokko, 1960-2010» [Pays d'origine : perspectives sur les liens avec le Maroc, 1960-2010], PhD, sous la dir. de Marlou Schrover, Leiden University.

—, 2019, "Vijf mythes over de komst van Marokkaanse gastarbeiders naar Nederland ontkracht» [Cinq idées reçues déconstruites sur l'arrivée des travailleurs immigrés aux Pays Bas], Trouw [https://www.trouw.nl/nieuws/vijfmythes-over-de-komst-van-marokkaanse-gastarbeiders-naar-nederlandontkracht $\sim$ be $74 \mathrm{~d} 721 /]$

CHIN Rita, 2007, The Guest Worker Question in Postwar Germany, Cambridge, Cambridge University Press.

COTTAar Annemarie, \& Nadia Bouras, 2009, Marokkanen in Nederland: de pioniers vertellen [Marocains aux Pays-Bas: les pionniers racontent], Amsterdam, J.M. Meulenhoff.

KeuZenkamp Saskia (ed.), 2010, Steeds gewoner, nooit gewoon. De Acceptatie van Homoseksualiteit in Nederland [De plus en plus commun, jamais ordinaire. L'acceptation de l'homosexualité aux Pays-Bas], Den Haag, Sociaal en Cultureel Planbureau. 
Mizrahi Sirazi [Mirdal] Gretty, 1970, «Tyrkerne », in Eggert PeTERSEN (ed.), Gestearbejder $i$ Kobenhavn: en undersogelse [Les travailleurs immigrés à Copenhague, une enquête] », Copenhagen, Mellemfolkeligt Samvirke.

SCHrover Marlou, 2011, Om de meisjes, voor de meisjes. Een historisch perspectief op problematisering en bagatellisering van onderwerpen die te maken hebben met migratie en integratie [Au sujet des filles, pour les filles. Perspective historique sur la problématisation et la banalisation des sujets liés à la migration et à l'intégration], Leiden, Leiden University.

SHADID W. [asef Abdel Rahman], 1979, "Moroccan Workers in the Netherlands », Ph.D, sous la dir. de J.D. SPECKMANN and J.P. VAN DE GEER, Leiden University.

SHIELD Andrew DJ, 2017, Immigrants in the Sexual Revolution: perceptions and participation in Northwest Europe, Cham (Switzerland), Palgrave Macmillan.

STOLER Ann Laura, 2010, Along the Archival Grain: epistemic anxieties and colonial common sense, Princeton, Princeton University Press.

Tuskan Erhan \& Jaap VogEL, 2004, Lied wit den vreemde: Brieven en foto's van Turkse migranten 1964-1976 [Chant de l'étranger: lettres et photos de migrants turcs 1964-1976], Amsterdam, Aksant. 